\title{
Práticas corporais e atividades físicas na perspectiva da Promoção da Saúde na Atenção Básica
}

\author{
Physical activity and corporal practices \\ from the perspective of Health Promotion in Primary Care
}

Fabio Fortunato Brasil de Carvalho ${ }^{1}$

Júlia Aparecida Devidé Nogueira ${ }^{2}$

${ }^{1}$ Escola Nacional de Saúde Pública Sergio Arouca, Fiocruz. R. Leopoldo Bulhões 1480, Manguinhos. 21041-210 Rio de Janeiro RJ Brasil. fabiofbcarvalho@gmail.com

${ }^{2}$ Faculdade de Educação Física, Universidade de Brasília. Brasília DF Brasil.

\begin{abstract}
The scope of this article is to make a critical reflection on the institutionalization and enhancement process of corporal practices and physical activity as Health Promotion actions in Primary Care in the Brazilian Unified Health System (SUS). To examine the configuration of this form of care and course of action, which has emerged and is becoming consolidated in public health, a brief historical review of the process of standardizing and implementing Health Promotion in the SUS was conducted, in order to reveal data on corporal practices and physical activity. Lastly, there is a critical analysis of interactions, interfaces and divergences arising between corporal practices and physical activities and the theoretical frameworks of Health Promotion. It is argued that part of the problem lies in the production of knowledge that overlooks the epistemological aspects of the relationship between corporal practices and physical activities. The conclusion drawn is that despite the exponential growth in the availability of these actions, it is still not possible to say that this form of care can be characterized as Health Promotion, as it would be necessary for some principles to be observed for this to occur, such as empowerment, social participation, autonomy, equity, completeness, among others.

Key words Public health, Unified health system, Health services
\end{abstract}

Resumo O presente artigo busca refletir criticamente sobre o processo de institucionalização e fortalecimento das práticas corporais e atividades físicas como ações de Promoção da Saúde na Atenção Básica do Sistema Único de Saúde (SUS). Para examinar a configuração dessa forma de cuidado e eixo de ação que emerge e vem se consolidando na saúde pública brasileira, optamos por revisitar historicamente, de forma sucinta, o processo de conformação e desenvolvimento da Promoção da Saúde no SUS de modo a, na sequência, apresentar alguns dados sobre as práticas corporais e atividades físicas nesta realidade. Por fim, há a análise crítica das interações, interfaces e distanciamentos que se processam entre as práticas corporais e atividades fisicas e os referenciais teóricos da Promoção da Saúde, em sua vertente crítica. Argumenta-se que parte do problema reside na produção de saberes que desconsideram os aspectos epistemológicos da relação entre práticas corporais e atividades físicas. Conclui-se que apesar do exponencial crescimento da oferta dessas ações, ainda não é possivel afirmar que esta forma de cuidado possa ser caracterizada como Promoção da Saúde já que seria necessário observar alguns princípios para isso, tais como empoderamento, participação social, autonomia, equidade, integralidade, entre outros.

Palavras-chave Saúde pública, Sistema Único de Saúde, Serviços de saúde 


\section{Introdução}

Múltiplos movimentos de fortalecimento da Atenção Básica (AB) e da Promoção da Saúde (PS) no Sistema Único de Saúde (SUS), assim como a ampliação das práticas corporais e atividades físicas (PC e AF) como um eixo de ação na saúde pública brasileira ocorreram nos últimos anos $^{1-3}$. Em 2016, os dez anos da publicação da Política Nacional de Promoção da Saúde (PNPS), um marco na institucionalização das PC e AF na $\mathrm{AB}^{3,4}$, e o exponencial crescimento da oferta desta forma de cuidado no cotidiano dos serviços ${ }^{5-7}$ justificam a reflexão crítica sobre o processo de institucionalização e implementação das PC e AF como ações de PS na AB.

Para isto é indispensável entender a relação que as $\mathrm{PC}$ e AF possuem com os princípios e vertentes ideológicas da $\mathrm{PS}$ na $\mathrm{AB}^{8,9}$. Assim sendo, através de revisão bibliográfica não sistemática apresenta-se uma breve retrospectiva sobre a conformação e consolidação do modelo de saúde pública brasileiro, destacando eventos marcantes na institucionalização e fortalecimento da PS e das PC e AF na AB. Os sistemas de busca utilizados foram a base de dados SciELO, Biblioteca Virtual em Saúde e sítios institucionais, em especial do Ministério da Saúde (MS), tendo como categorias-chave as PC e AF, PS, AB e correlatos. Também foi realizado contato com o MS para a disponibilização de dados.

$\mathrm{Na}$ sequencia, analisa-se alguns dados secundários sobre a implementação das $\mathrm{PC}$ e $\mathrm{AF}$ no SUS obtidos através da avaliação do Programa Nacional de Melhoria do Acesso e da Qualidade da Atenção Básica (PMAQ), do monitoramento do Programa Academia da Saúde e de pesquisas realizadas sobre o tema. Por fim, realizou-se análise crítica sobre as configurações e relações que permeiam a institucionalização e a implementação das PC e AF e a PS na AB nos dez anos seguintes à aprovação da PNPS.

\section{A institucionalização e o fortalecimento das Práticas Corporais e Atividades Físicas e da Promoção da Saúde na Atenção Básica}

Debates contemporâneos sobre o conceito de saúde se iniciam no Brasil por volta de 1970 e se intensificam a partir de 1980 com o processo de redemocratização do país ${ }^{1}$. Em consonância com as amplas discussões sobre o modelo de atenção à saúde o SUS adotou o conceito ampliado de saúde, que envolve modos de ser e produzir e/ou recriar a vida em sua singularidade, multidimen- sionalidade e sustentam a expansão da cobertura à saúde através de um sistema público centrado na $\mathrm{AB}^{10,11}$ em consonância com o que foi discutido em Alma-Ata na Conferência Internacional sobre Cuidados Primários de Saúde, em 1976 ${ }^{12}$. Concomitantemente, discussões emergentes sobre PS passam a destacá-la como um modelo teórico-conceitual capaz de subsidiar políticas e ações governamentais e de servir como eixo orientador das práticas em saúde pública ${ }^{1,2}$.

Em 1990 é criado o Programa Saúde da Família, primeiro programa a se inspirar e operar com preceitos de PS, depois transformado em Estratégia (ESF), a qual é a principal aposta de reorientação do modelo assistencial em saúde ${ }^{10}$. Organizada em equipes multiprofissionais que atuam nas Unidades Básicas de Saúde (UBS) e no território, a ESF valoriza as ações de promoção e proteção da saúde, prevenção das doenças e atenção integral às pessoas no ambiente em que vivem, favorecendo a compreensão ampliada do processo saúde/doença ${ }^{10,11}$.

A partir de 1998, o fortalecimento de cooperações internacionais e o sucesso de alguns programas nacionais (e.g. o controle do tabagismo) favorecem a introdução formal do tema da PS nos debates sobre a saúde ${ }^{1}$ e culminam com a redação de um documento inicial da PNPS, em $2002^{12}$, que nunca teve real vigência no interior do SUS ${ }^{1}$. Nesse período, premido pelo panorama epidêmico das Doenças Crônicas Não Transmissíveis (DCNT) e por inciativas internacionais (e.g. Estratégia Global para Alimentação Saudável, Atividade Física e Saúde da Organização Mundial de Saúde - EG), o MS desenvolveu alguns documentos (e.g. cartilha "A construção de vidas mais saudáveis"), projetos (e.g. Agita Brasil: Programa Nacional de Promoção da Atividade Física e Programa Pratique Saúde) e editais de financiamento (de 2005 a 2010) que incluíam o desenvolvimento de ações relativas às $\mathrm{PC}$ e $\mathrm{AF}^{1,3-5}$.

O ano de 2006 inclui a publicação de marcos normativos importantes que enfatizam a PS na $\mathrm{AB}$ e que, de forma direta ou indireta, apresentam relação com as PC e AF, são: a Política Nacional de Atenção Básica (PNAB), a Política Nacional de Práticas Integrativas e Complementares (PNPIC); e a própria PNPS. A PNPS reforça o papel dos determinantes sociais no processo saúde-doença e inclui as PC e AF como uma de suas ações prioritárias ${ }^{9}$. A PNPIC integra outros sistemas médicos e recursos terapêuticos ao SUS na perspectiva da PS e prevenção de agravos, voltada ao cuidado continuado, humanizado e integral em saúde, incluindo serviços de $\mathrm{PC}$ e $\mathrm{AF}^{13}$. Por 
sua vez, a PNAB reafirma a ESF como a estratégia prioritária para organizar a $\mathrm{AB}$, favorecendo o acesso universal e contínuo a serviços de saúde de qualidade mediante o cadastramento e a vinculação dos usuários ${ }^{10}$, aspecto reforçado pela atual PNAB, publicada em $2011^{11}$.

Outro marco no processo de consolidação da $\mathrm{AB}$ e fortalecimento das PC e AF é a criação, em 2008, dos Núcleos de Apoio à Saúde da Família (Nasf), possibilitando a ampliação da oferta, da abrangência e da resolutividade dos serviços de saúde na rede através de equipes multiprofissionais, inclusive profissionais de Educação Física, de forma integrada com a ESF, o que favoreceu o fortalecimento das PC e AF como oferta de cuidado $^{14-16}$

Os avanços e os desafios impostos pelas transformações sociais, o acúmulo de reflexões teórico-conceituais, as atualizações e as inovações nas práticas, e a necessidade de articulação entre diferentes áreas técnicas, programas e políticas faz com que o MS tome uma série de medidas que fortalecem o papel e a importância da $\mathrm{AB}$ no conjunto das políticas e programas de saúde pública ${ }^{11}$. Em 2011, publica uma versão aprimorada da PNAB, aumenta o financiamento da $\mathrm{AB}$ em mais de $100 \%$ entre 2010 e 2014, promove a estratégia de ampliação e qualificação da infraestrutura das UBS (Requalifica UBS), lança o PMAQ e o Programa Academia da Saúde, dentre outras ações ${ }^{11}$.

Na perspectiva das PC e AF merece destaque, além do Nasf, o Programa Academia da Saúde, uma das iniciativas constantes no Plano de Ações Estratégicas para Enfrentamento das DCNT no Brasil de 2011 a $2022^{17}$, para aumentar a AF na população, oferecendo polos com infraestrutura, equipamentos e quadro de pessoal qualificado para a orientação de PC e AF, de lazer e modos de vida saudáveis ${ }^{4,17}$.

Em 2013, o Programa Academia da Saúde teve sua normativa revista e seus objetivos ampliados para favorecer a PS e a produção do cuidado e de modos de vida saudáveis e sustentáveis da população através de PC e AF, alimentação saudável, da ampliação da autonomia dos indivíduos sobre as escolhas de modos de vida saudáveis; do aumento do nível de AF da população, entre outros ${ }^{17}$.

Em 2014, a PNPS revista é publicada. Elaborada coletivamente a partir de múltiplos movimentos simultâneos como estratégias de escuta, mobilização e produção de sínteses, avança-se no diálogo com gestores, trabalhadores e movimentos sociais, conseguindo estabelecer formas de articulação mais permanentes e sustentáveis na medida em que nessa relação podem se multiplicar possibilidades de enfrentamento dos problemas de saúde pública e qualificar cada vez mais as ações no SUS $^{18}$.

\section{Panorama atual das Práticas Corporais e Atividades Físicas na Atenção Básica}

A seguir serão apresentados alguns dados sobre o desenvolvimento das $\mathrm{PC}$ e $\mathrm{AF}$ na $\mathrm{AB}$ bem como alguns elementos relacionados ao tema. Destacamos que não há a pretensão de realizar uma revisão sistemática da literatura nem tampouco de esgotar todas as dimensões dessa complexa análise no presente artigo. Os dados reportados visam ilustrar o panorama atual com vistas a subsidiar uma reflexão crítica sobre a essência das PC e AF realizadas no contexto da $\mathrm{AB}$, tendo a PS como base conceitual.

A despeito de encontrarmos alguns registros de iniciativas referentes às $\mathrm{PC}$ e $\mathrm{AF}$ e a presença do Profissional de Educação Física no SUS a partir dos anos $2000^{16,17,19}$, principalmente relacionados a grupos de caminhadas através do projeto para hipertensos e diabéticos conhecidos como Hiperdia $^{16}$ e outras iniciativas municipais a partir de $2005^{3}$, é inegável que o lançamento da PNPS em 2006 e os subsequentes apoios técnico-financeiros do gestor federal alavancaram de forma marcante a institucionalização das PC e AF no SUS ${ }^{4}$.

Edital inaugural para financiamento, pelo MS, de ações consonantes com a EG em 2005 contemplou as 27 capitais e o Distrito Federal ${ }^{3}$. A partir de 2006 o edital foi aberto a todos os municípios e versou sobre as ações específicas da PNPS, havendo grande difusão de iniciativas relacionadas às $\mathrm{PC}$ e AF ${ }^{4}$. Em 2008 foram 523 e em 2009 foram 1.254 entes federados contemplados com repasse financeiro. Até o início de 2011 haviam sido transferidos recursos financeiros para implementação das ações da PNPS em 1.465 municípios, dos quais $73,7 \%$ informaram realizar ações de $\mathrm{PC}$ e $\mathrm{AF}^{4}$.

Monitoramento realizado pelo MS, com 255 entes federados financiados até 2008, mostrou que a maioria dos projetos, $88,5 \%$, contemplava as $\mathrm{PC}$ e AF, $94 \%$ estavam vinculados à ESF, sendo que $35,1 \%$ desenvolviam atividades em parceria com os Nasf e utilizando predominantemente estruturas como praças/parques, espaços de caminhada e UBS para desenvolver as práticas ${ }^{5}$. Atualmente, a maioria das ações e programas de PC e AF no SUS são desenvolvidos através do Nasf e do Programa Academia da Saúde ${ }^{4}$. Ambos 
possibilitam a inclusão do Profissional de Educação Física nas equipes, favorecendo a oferta dessas práticas como cuidado em saúde $e^{15,16,20-22}$. Destaca-se que, a depender contexto e objetivo das PC e AF, é possível que diferentes categorias profissionais atuem com tais atividades no SUS, contudo é inegável a aproximação daqueles profissionais com as $\mathrm{PC}$ e $\mathrm{AF}^{20}$.

Assim, dados do PMAQ e do monitoramento anual do Programa Academia da Saúde fornecem um panorama situacional quantitativo das PC e AF na $A B$, além de apresentar alguns fatores contextuais relacionados. Das equipes de AB participantes do $2^{\circ}$ ciclo do PMAQ, 96\% (n $=28.579$ ) afirmam ofertar ações educativas e de PS, das quais,respectivamente, $44,2 \%$ incentivam e desenvolvem PC e $68,8 \%$ desenvolvem AF6 nas UBS e/ou no território (no instrumento de avaliação as PC e AF estão separadas nas opções de resposta), evidenciando a oferta de PC e AF também pela ESF, além do Nasf e do Programa Academia da Saúde.

No Nasf, são aproximadamente 2.500 Profissionais de Educação Física distribuídos em mais de 4.000 equipes em cerca de 3.300 municípios ${ }^{6}$. Estudo realizado até 2011 mostra que este profissional estava entre as cinco profissões mais contratadas para trabalhar no Nasf, estando presente, em média, em $49,2 \%$ das equipes, mas com diferenças expressivas entre regiões (e.g. 75\% no Acre e Paraná e $0 \%$ no Distrito Federal) ${ }^{15}$.

Há ainda cerca de 4.000 polos do Programa Academia da Saúde implantados ou em processo de implantação em 2.800 municípios ${ }^{6}$. Dados do monitoramento do Programa em 2015 com 2.418 respondentes indicam que dos 859 polos em funcionamento, $96 \%$ oferecem ações no eixo das PC e AF, 96\% alimentação saudável, 94\% educação em saúde, $88 \%$ mobilização da comunidade, $57 \%$ práticas integrativas e complementares e $27 \%$ práticas artísticas ${ }^{7}$. Não obstante, $47 \%$ dos respondentes no monitoramento do Programa informaram dificuldades para a contratação de profissionais ${ }^{7}$, trazendo à tona outra questão relevante: a precarização e a flexibilização dos contratos de trabalho, o que pode resultar em baixa estabilidade, na criação de outros vínculos empregatícios e na redução da qualidade do serviço ${ }^{15}$.

A despeito da grande parte dos 859 polos do Programa Academia da Saúde em funcionamento estar em municípios que possuem equipe do Nasf (77\% dos respondentes), e de estar próximos à UBS ( $89 \%$ dos respondentes), o que deveria potencializar a articulação e a complementação das atividades e favorecer o cuidado amplia- do, $24 \%$ dos respondentes relataram dificuldades na articulação com a ESF e 20\% com o Nasf'. Em outras realidades analisadas houve relatos de dificuldade para utilização de salas nas UBS, falta de espaço físico e de materiais ${ }^{15,16,23}$.

Ademais, os dados indicam que $90 \%$ dos polos respondentes atendem apenas a comunidade adscrita, e um baixo percentual, aproximadamente $3 \%$, indicou a inclusão de populações específicas (e.g. quilombolas, ribeirinhos, indígenas, pessoas em situação de rua e ciganos), sendo que apenas 5\% afirmaram não haver nenhum desses grupos no município ${ }^{7}$.

Quanto ao público alvo do programa, quase a totalidade dos polos desenvolvem atividades com adultos e idosos, $76 \%$ incluem adolescentes, $38 \%$ atendem crianças, e apenas $36 \%$ possuem participantes em todas as faixas etárias. Em adição, $56 \%$ dos polos informam ter dificuldades para desenvolver atividades com crianças, $41 \%$ com adolescentes e $43 \%$ dos polos têm encontrado dificuldades para envolver a comunidade no planejamento de atividades ${ }^{7}$. Esses dados são corroborados por outros estudos que apontam o perfil da população usualmente engajada nessas práticas: adultos e idosos, do gênero feminino e com alguma patologia ${ }^{15,16,23}$.

Estudos complementares indicam que as PC e $\mathrm{AF}$ mais desenvolvidas na $\mathrm{AB}$ são as aeróbicas (principalmente caminhada) e os exercícios resistidos e, em menor proporção, o alongamento, a coordenação motora e as atividades lúdi$\operatorname{cas}^{4,5,15,16,23-27}$, o que, para alguns autores, pode evidenciar a difusão de estilos de vida saudável numa abordagem comportamental e de forte perspectiva curativa ${ }^{9,13,28-30}$.

\section{Problematizando as Práticas Corporais e Atividades Físicas na perspectiva da Promoção da Saúde}

A despeito do exponencial crescimento da oferta de PC e $A F$ na $A B^{4,6,7}$ e das evidências de seus benefícios à saúde individual e coletiva ${ }^{31}$, é essencial problematizar a generalização de que as PC e AF na AB são, necessária e aprioristicamente, ações de PS. É indispensável analisar a que vertentes político-ideológicas as $\mathrm{PC}$ e $\mathrm{AF}$, recomendadas nos documentos e oferecidas na prática, têm se filiado nos últimos anos. Isso porque a institucionalização e a implementação das PC e AF enquanto serviço de saúde pública também são marcadas pelas fortes disputas político-ideológicas presentes na instituição e no fortalecimento do SUS e da PS ${ }^{1,2,8,9}$. 
Não obstante o avanço em publicações e experiências que entendem as PC e AF como uma forma de cuidado ampliado desenvolvido consoante os princípios modernos de PS, é a concepção limitada da relação entre PC e AF e PS que transita hegemonicamente na grande área da saúde. Grande parte dos conteúdos de formação profissional, de artigos científicos, e das políticas, normativas e práticas em saúde pública compartilham uma compreensão estatística de que as PC e AF são promotoras de benefícios biológicos à saúde individual - em geral expressos por alguma medida de aptidão física.

Sem desconsiderar os achados das ciências naturais, é importante que as análises referentes às $\mathrm{PC}$ e AF como elemento de PS busquem avançar para uma profícua interlocução com as ciências sociais e humanas na perspectiva de ampliar a compreensão dos fenômenos numa perspectiva crítica. Em tempo, vale lembrar as discussões sobre biopoder empreendidas pelo campo da saúde coletiva ${ }^{28}$ e que a formação do Profissional de Educação Física, a despeito dos incontestáveis avanços críticos aportados pelas ciências da educação, advém de uma tradição de adequar os corpos, através de disciplina militar, para que estes estejam aptos a movimentar os meios de produção em uma sociedade que vivia o desenvolvimento industrial ${ }^{13,15,22,29}$.

Adotar uma perspectiva crítica implica em estabelecer o marco epistemológico no processo de produção do conhecimento. É bastante comum que os termos "promoção da saúde" ou "prevenção de doenças", e "atividade física” ou "práticas corporais" sejam utilizados no cotidiano leigo, científico e/ou político como se fossem sinônimos. Não obstante, cada um dos termos mantém especificidades ligadas a visões de mundo e projetos de sociedade que os tornam diferentes e conflitantes $^{9,32}$. O uso de cada um dos conceitos pode representar a adoção de uma abordagem que se expressa nas formas de conceber e organizar os discursos e as práticas relativas aos seus saberes e conhecimentos ${ }^{33}$.

Limites conceituais, controvérsias e ambiguidades nas diversas abordagens sobre PS e prevenção de doenças ressaltam a diferença de ênfases e entendimentos presentes em debates científicos, documentos institucionais e práticas em serviço ${ }^{8,9}$. A vertente crítica da PS extrapola a perspectiva reducionista de que PC e AF são ações que estão sob governabilidade total de sujeitos, ou seja, que são simples de serem adotadas como práticas de vida e defende alguns princípios, tais como participação social e autonomia, que devem perpassar a perspectiva promotora de saúde ${ }^{1}$.

No mesmo sentido os termos PC e AF vão sendo diferenciados, expondo os tensionamentos entre o paradigma biologizante da aptidão física e o conceito de risco subjacente ao discurso vigente sobre o sedentarismo ${ }^{28,33}$, e o caráter cultural e lúdico do fenômeno com suas várias ligações sociais ${ }^{32,33}$.

Embora haja publicações que tentam relativizar tal compreensão, as ambiguidades epistemológicas que acreditamos existir na literatura entre PS e prevenção de doenças; e entre PC e AF não serão aprofundadas neste texto por limitações de espaço. No presente artigo optamos pela utilização da terminologia conjunta PC e AF como aparece na PNPS, ainda que tal decisão possa atenuar diferenças e suscitar simplificações conceituais. No entanto, os termos podem aparecer separados quando se tratar de citação, respeitando a opção dos autores. Para informações adicionais sugerimos a leitura de Carvalho ${ }^{20}$, Lazzarotti Filho et al. ${ }^{34}$ e Damico e Knuth ${ }^{35}$.

Para avançar nessa reflexão, é importante reconhecer que a AF é devidamente pautada nos debates sobre saúde pública ${ }^{3-5}$ a partir da medida de indicadores epidemiológicos que permitiram traçar um contexto de mudanças sociossanitárias - tais como as transições epidemiológica, demográfica, nutricional - e o acúmulo de evidências demonstrando a associação entre AF com a manutenção ou melhora da aptidão física, representando um fator de proteção às $\mathrm{DCNT}^{31}$. A ideia de promover estilos de vida saudáveis e ativos como estratégia de saúde pública de forma simplista encontra eco nas abordagens preventivistas e conservadoras ou comportamentalistas de PS, como expresso no relatório Lalonde, publicado em 1974, no qual o enfoque das ações é voltado para a redução dos fatores de risco com ênfase na ação individual ${ }^{28}$.

Cabe apontar que não realizaremos o resgate histórico do conceito de PS, Westphal ${ }^{36}$, entre outros, apresenta tal resgate. Ideias que se aproximam do que foi construído a partir da Carta de Ottawa remontam do século XVIII. Aqui destacamos a época citada por estar relacionada ao paradigma da AF e aptidão física como fator de proteção à saúde.

Organizações nacionais e internacionais de saúde passam então a endossar e divulgar recomendações de base populacional para a prática de AF (ou exercícios) como um fator de proteção à saúde respaldadas por evidências científicas centradas em marcadores biológicos, tais como a 
aptidão física ${ }^{31}$. A ciência moderna utiliza hegemonicamente o arcabouço teórico-metodológico da epidemiologia ${ }^{31}$ para produzir as evidências de vinculação entre as $\mathrm{PC}$ e $\mathrm{AF}$ e a saúde.

Essa perspectiva dominante e pretensamente unívoca sobre as PC e AF e saúde incorre em simplificações na compreensão dos fenômenos de forma que as práticas de saúde, descontextualizadas, passam a responsabilizar e culpabilizar o sujeito vulnerável por seu adoecimento. Em adição, a falta de rigor metodológico nas reflexões associativas e nas questões epistemológicas vem gerando resultados e compreensões equivocadamente deterministas dos fenômenos das PC e $\mathrm{AF}$ e da PS, contribuindo para a manutenção da visão positivista, a banalização do tema e a perpetuação do senso-comum ${ }^{32,33}$.

Não obstante, coexistindo com esses movimentos, debates da I Conferência Internacional de PS, publicados na Carta de Ottawa em 1986, resgatam a concepção de produção social da saúde com perspectivas contextuais amplas, históricas e coletivas e apontam que a PS deve buscar atuar sobre os fatores do entorno social, as condições de vida e de trabalho, as condições culturais, ambientais, entre outras, por meio de políticas públicas e ações de âmbito comunitário e universal $^{1,2}$. Se relaciona ao esforço planejado para construir políticas públicas saudáveis, criar ambientes favoráveis, fortalecer a ação comunitária, desenvolver habilidades pessoais e/ou reorientar serviços de saúde na busca de metas e devem, inequivocamente, incluir aspectos sociais, culturais e econômicos ${ }^{37}$. Essa compreensão evidencia o papel dos determinantes sociais no processo de construção das condições de vida e saúde de indivíduos e coletividades ${ }^{33}$ e demarca a concepção moderna ou crítica de PS ${ }^{1}$.

Nessa perspectiva, é importante nos deter na PNPS, principal marco normativo da institucionalização das PC e AF na AB. A PNPS de 2006, gestada no seio da Secretaria de Vigilância em Saúde do MS foi marcadamente influenciada pela $\mathrm{EG}$, na qual o destaque conferido às $\mathrm{PC} \mathrm{e}$ AF na saúde decorre de constatações epidemiológicas sobre seus benefícios frente às doenças do aparelho circulatório e pela compreensão de que a inatividade física é um dos principais fatores de risco para a mortalidade mundial ${ }^{4,9}$.

De forma similar às contradições presentes na PNPS de $2006^{8,9}$, as práticas de saúde na $\mathrm{AB}$ ainda buscam superar o modelo assistencial vigente, calcado na supervalorização da assistência curativa, especializada e hospitalar, que induz ao excesso de procedimentos tecnológicos e medica- mentosos e, sobretudo, à fragmentação do cuida$\mathrm{do}^{11,37}$. O mesmo ocorre com as PC e AF, em que a concepção e o escopo de cada programa implicam no planejamento e na execução de ações e atividades com foco, objetivos e qualidade distintas, em se tratando da concepção de saúde e de PS subjacentes ${ }^{38,39}$.

As PC e AF podem ser concebidas como ferramentas da PS moderna ou crítica, voltadas para ações integradoras e descentralizadas que contam com ampla participação social para melhorar as condições vida da populaçãa ${ }^{33}$, mas também podem ser vistas como ações simples de serem adotadas, nas quais sujeitos e coletividades, por vontade e esforço próprios, poderiam começar a praticá-las a qualquer momento, em qualquer lugar, desconsiderando o contexto de vida no qual estão inseridos.

Ainda é comum que práticas nomeadas como de PS sejam desenvolvidas centradas em discursos prescritivos e antecipatórios de riscos à saúde, numa perspectiva biomédica, individual, que acaba na responsabilização por escolhas e hábitos de vida ${ }^{37-40}$. Essa perspectiva reducionista desconsidera os modos de produção da existência humana, abordados historicamente como fenômenos constituintes, produtores, reprodutores ou transformadores das relações sociais e da própria saúde, e os conceitos envolvidos na experiência concreta da saúde e do adoecer ${ }^{13,32,33}$.

A problematização apresentada dialoga com reflexões sobre o próprio desenvolvimento da racionalidade científica e a emergência dos campos científicos, em especial da medicina, e por conseguinte da saúde pública ${ }^{40} \mathrm{e}$ da educação física, derivados do método experimental e da tradição quantitativa de pesquisa, que deixam como legado a fragmentação e a neutralização de dimensões subjetivas fundamentais ao estudo da vida, da saúde, do corpo e de suas relações com o movimento humano ${ }^{29}$.

O discurso do risco é fortalecido e naturalizado por estudos e monitoramentos que, ao adotarem um âmbito mais técnico, centrado nas ciências naturais, destacam a operacionalização do método em detrimento das reflexões acerca das relações humanas em saúde mediadas pelo movimento corporal, e acabam por desconsiderar a natureza multidimensional desses fenômenos ${ }^{41}$. Tal concepção de ciência tem um óbvio impacto na definição e na produção de saúde, vinculando -as hegemonicamente ao seu aspecto biológico e, sobre as PC e AF, fixando-se no seu desfecho: a aptidão física ${ }^{32}$. Vale destacar que esse conhecimento científico também embasa diretrizes e posiciona- 
mentos oficiais que norteiam a formação profissional e, consequentemente, as práticas em saúde.

A renúncia ao rigor filosófico e, por conseguinte, metodológico, na análise destes conceitos centrais à institucionalização de práticas promotoras de saúde tem produzido noções que se limitam às visões do indivíduo isolado de sua cultura $^{28}$. Olhares simplistas e acríticos hegemonicamente centrados em saberes epidemiológicos, clínicos e/ou biológicos não são capazes de, sozinhos, explicarem relações entre fenômenos complexos como o movimento corporal e a saúde humana, muito menos de promoverem saúde de forma descontextualizada da realidade social ${ }^{41}$.

Destacamos que esta reflexão não invalida ou nega a necessidade de se considerar e incluir saberes epidemiológicos e clínicos nas práticas de diferentes campos profissionais atuantes na PS, uma vez que têm importância e utilidade notoriamente reconhecidas. A epidemiologia é um campo de conhecimento necessário para o âmbito das análises e intervenções sanitárias, mas está longe de ser suficiente, como, por vezes, parece se autorizar a assumir tal papel ${ }^{33,41}$.

É necessário considerar que a perspectiva epidemiológica dos fatores de risco, fundante na relação entre as PC e AF e a saúde, subsidia protocolos discursivos relacionados ao mal do sedentarismo, que interferem objetivamente e subjetivamente nas decisões acerca das práticas cotidianas $^{28}$. Propostas conservadoras de PS baseadas em modelos comportamentais agrupados pela ideia do estilo de vida saudável acabam por utilizar estratégias que suscitam a culpabilização preventiva daqueles que se expõem aos riscos tecnicamente pré-dimensionados, fortalecendo a dimensão persecutória aos indivíduos ${ }^{33,40}$ e o uso do biopoder ${ }^{28,33}$.

O discurso da vida ativa dá, a priori, uma aura positiva para a AF, o que facilita sua aceitação e dificulta críticas, pois, independentemente de qualquer observação ou análise, ninguém deveria ser contrário a algo tão genuinamente saudável ${ }^{33,40}$. Em adição, dada a ampla possibilidade de compreensões, o uso irrefletido, indiscriminado e confuso de conceitos relacionados à PS em produções científicas, políticas públicas e serviços em saúde favorece a aproximação apriorística entre elas e as $\mathrm{PC}$ e $\mathrm{AF}^{33}$.

Torna-se imprescindível pensar as $\mathrm{PC}$ e AF na atual dinâmica social, cultural e econômica e suas amplas intersecções, principalmente na sua função de afirmar, confirmar e/ou reconstruir a hegemonia de um projeto histórico-político ${ }^{41}$. É imperioso superar o modelo de valorização do movimento humano como fator de PS na perspectiva exclusiva da aptidão física, da pretensa longevidade com vitalidade e do melhor ajuste do homem à sociedade capitalista. Faz-se necessário afastar a perspectiva neohigienista, de hiperprevenção, com forte conteúdo moral e normativo, e produzir sentido com as PC e AF para que elas se tornem efetivamente promotoras de saúde ${ }^{28,33}$.

A despeito de avanços recentes, tanto a esfera conceitual quanto a realidade dos serviços ainda são fortemente influenciadas por monitoramentos e pesquisas com abordagens epidemiológicas tradicionais, preventivistas, biológicas e comportamentais $^{31,32}$. Algumas vertentes das ciências da saúde, em interlocução com as ciências sociais e humanas, buscam refletir criticamente sobre ambiguidades e imprecisões epistemológicas quanto aos termos e conceitos PC e AF, e PS e prevenção de doenças presentes tanto em artigos científicos quanto em documentos governamentais, que se refletem nas práticas em saúde ${ }^{41}$.

É importante reconhecer a crescente relevância das PC e AF voltadas para a saúde como um fato social complexo presente na vida cultural contemporânea $^{13}$. Contudo, não há mais como continuar afirmando genericamente que as $\mathrm{PC} \mathrm{e}$ AF promovem saúde e bem-estar sem examinar de forma mais criteriosa as argumentações ideológicas acríticas que ocultam a mercantilização não só da saúde, mas da cultura corporal e da própria vida humana ${ }^{33}$. São necessários mais estudos de caráter qualitativo para compor análises ampliadas sobre as relações entre as PC e AF e a saúde, trazendo para o debate científico elementos que devem permear a formação profissional e a atuação em programas e politicas públicas de PS.

Propostas modernas de PC e AF a partir da PS devem valorizar as experiências dos sujeitos em seus contextos social, familiar, cultural e biológico, além das subjetividades criadas a partir delas ${ }^{20}$ e as práticas devem tentar contemplar valores e princípios de PS, como exposto na versão revisada da PNPS ${ }^{18}$. Em conformidade com essa concepção e com os princípios do SUS, as PC e AF ofertadas na $\mathrm{AB}$ devem buscar fomentar a autonomia, o empoderamento e a participação social, as potencialidades relacionadas à construção de vínculo com os usuários ao propiciar a ludicidade e o encontro, a criação e o compartilhamento de objetivos coletivos, entre outros, o que muitas vezes pode não ser possível na prática clínica ${ }^{42}$. Precisam ainda ser valorizadas e associadas à uma perspectiva participativa voltada para a melhora das condições de vida e saúde e o desenvolvi- 
mento integral das habilidades e capacidades de crianças, jovens, adultos e idosos e a redução das iniquidades por meio da interação entre a família, a comunidade local e a sociedade ${ }^{37,43}$.

Adotar modos de vida saudável dos quais as PC e AF fazem parte não deve ser compreendido como uma decisão individual simples, nem decorrente de soluções simplistas ${ }^{27}$. Realizar, ou não, as $\mathrm{PC}$ ou $\mathrm{AF}$ envolve fatores mais amplos como a disponibilidade de locais, de tempo e de recursos apropriados, e da similitude entre a oferta das atividades e o interesse pessoal, dentre outros. Assim como a PS, a construção e o fortalecimento de modos de vida saudável e da qualidade de vida para indivíduos e coletividades é complexa e processual ${ }^{42}$.

\section{Considerações finais}

Após dez anos da publicação da PNPS, o resgate histórico e a análise crítica empreendida sobre as relações e configurações que permeiam o processo de institucionalização das PC e AF como ações de $P S$ na $A B$ mostram que: i) houve expressivo aumento na oferta das PC e AF em diferentes serviços e programas da $\mathrm{AB}$ favorecendo $\mathrm{o}$ acesso a essas práticas; ii) a visão das $\mathrm{PC}$ e $\mathrm{AF}$ apenas como fator de prevenção de DCNT, baseada nos saberes da biologia e da epidemiologia, ainda é hegemônica em pesquisas científicas, políticas, programas e práticas de saúde pública, representando um desafio a ser superado; iii) é necessário que as equipes de saúde da $\mathrm{AB}$ : ESF, Nasf, do Programa Academia da Saúde, entre outras, avancem na oferta das PC e AF numa perspectiva ampliada a partir da multidisciplinariedade, aumentando o escopo e as modalidades oferecidas, a participação social e o acesso de populações menos presentes na $\mathrm{AB}$, tais como adolescentes e homens; e, iv) lentamente avançamos para a ampliação da compreensão das PC e AF como potentes ferramentas de PS em sua perspectiva moderna e crítica, reconhecendo que essas práticas favorecem a obtenção de benefícios biológicos à saúde individual, mas que também são capazes de fortalecer a equidade, a autonomia, o empoderamento e protagonismo do sujeito, além de contribuir para a integralidade e sustentabilidade do cuidado no sistema de saúde.

\section{Colaboradores}

FFB Carvalho e JAD Nogueira contribuíram igualmente para a concepção do artigo, realizaram análise e interpretação das informações e dados, redigiram e revisaram criticamente o conteúdo e aprovaram a versão final a ser publicada.

\section{Agradecimentos}

Aos Departamentos de Atenção Básica (DAB/ SAS) e de Vigilância de Doenças e Agravos Não Transmissíveis e Promoção da Saúde (DEVDANTPS/SVS) do Ministério da Saúde pela cessão de dados. 


\section{Referências}

1. Buss PM, Carvalho AI. Desenvolvimento da promoção da saúde no Brasil nos últimos vinte anos (1988-2008). Cien Saude Colet 2009; 14(6):2305-2316.

2. Castro A, Malo M, organizadores. SUS: ressignificando a promoção da saúde. São Paulo: Hucitec, OPS; 2006.

3. Malta DC, Castro AMD, Gosch CS, Cruz DKA, Bressan A, Nogueira JD, Morais Neto OL, Temporão JG. A Política Nacional de Promoção da Saúde e a agenda da atividade física no contexto do SUS. Epidemiol Serv Saúde 2009; 18(1):79-86.

4. Malta DC, Silva MMA, Albuquerque GM, Amorim RCA, Rodrigues GBA, Silva TS, Jaime PC. Política Nacional de Promoção da Saúde, descrição da implementação do eixo atividade física e práticas corporais, 2006 a 2014. Rev Bras Ativ Fis Saúde 2014; 19(3):286-299.

5. Knuth AG, Malta DC, Cruz DKA, Freitas PCD, Lopes MP, Fagundes J, Reis RS, Hallal PC. Rede nacional de atividade física do Ministério da Saúde: resultados e estratégias avaliativas. Rev Bras Ativ Fís Saúde 2010; 15(4):299-233.

6. Brasil. Ministério da Saúde (MS). Departamento de Atenção Básica. Dados do Programa Nacional de Melhoria do Acesso e da Qualidade da Atenção Básica (PMAQ) e da gestão da Atenção Básica. Brasília: MS; 2015.

7. Brasil. Ministério da Saúde (MS). Departamento de Doenças e Agravos Não Transmissíveis e Promoção da Saúde. Monitoramento anual do Programa Academia da Saúde. Brasília: MS; 2015.

8. Traverso-Yèpez MA. Dilemas na promoção da saúde no Brasil: reflexões em torno da política nacional. Interface (Botucatu) 2007; 11(22):223-238.

9. Cruz DKA. Da promoção à prevenção: o processo de formulação da política nacional de promoção da saúde no periodo de 2003 a 2006 [dissertação]. Recife: Fundação Oswaldo Cruz; 2010.

10. Andrade LOM, Barreto ICHC, Bezerra RC. Atenção primária à saúde e estratégia saúde da família. In: Campos GWS, Minayo MCS, Akerman M, Drumond Júnior M, Carvalho YM, organizadores. Tratado de Saúde Coletiva. São Paulo, Rio de Janeiro: Hucitec, Abrasco; 2006. p. 783-836.

11. Magalhães Júnior HM, Pinto HA. Atenção Básica enquanto ordenadora da rede e coordenadora do cuidado: ainda uma utopia? Revista Divulgação em Saúde para Debate 2014; 51:14-29.

12. Brasil. Ministério da Saúde (MS). Secretaria de Políticas de Saúde. Projeto Promoção da Saúde. As Cartas da Promoção da Saúde. Brasília: MS; 2002.

13. Luz MT. Novos saberes e prática em saúde coletiva: estudo sobre racionalidades médicas e atividades corporais. São Paulo: Hucitec; 2005.

14. Florindo AA. Núcleos de Apoio à Saúde da Família e a promoção das atividades físicas no Brasil: de onde viemos, onde estamos e para onde vamos. Rev Bras Ativ Fís Saúde 2012; 14(2):72-73.

15. Santos SFS. Núcleo de apoio à Saúde da Família no Brasil e a atuação do profissional de Educação Física[dissertação]. Florianópolis: Universidade Federal de Santa Catarina; 2012.

16. Martinez JFN, Silva AM, Silva MS. As diretrizes do NASF e a presença do profissional em Educação Física. Motrivivência 2014; 26(42):207-221.
17. Malta DC, Silva Júnior JB. O plano de ações estratégicas para o enfrentamento das doenças crônicas não transmissíveis no Brasil e a definição das metas globais para o enfrentamento dessas doenças até 2025: uma revisão. Epidemiol Serv Saúde 2013; 22(1):151-164.

18. Rocha DG, Alexandre VP, Marcelo VC, Rezende R, Nogueira JAD, de Sá RF. Processo de revisão da Política Nacional de Promoção da Saúde: múltiplos movimentos simultâneos. Cien Saude Colet 2014; 19(11):43134322.

19. Lucena D, Borges KEL, Aguiar MA, Alcântara F, Madruga JG, Lomeo R. A Inserção da Educação Física na Estratégia Saúde da Família em Sobral/CE. Sanare 2004; 5(1):87-91.

20. Carvalho FFB. Práticas corporais e atividades físicas na Atenção Básica do Sistema Único de Saúde - ir além da prevenção das doenças crônicas não transmissíveis é necessário. Movimento 2016; 22(2):abr./jun. (publicação avançada)

21. Guarda FRBD, Silva RND, Araújo Júnior JLDACD, Freitas MIDF, Neto S. Intervenção do profissional de educação física: formação, perfil e competências para atuar no Programa Academia da Saúde. Rev Pan-Amaz Saúde 2014; 5(4):63-74

22. Bennedetti TRB, Silva DAS, Silva KS, Nascimento JV, organizadores. A formação do profissional de Educação Física para o setor saúde. Florianópolis: Postmix; 2014.

23. Silva ALF, Sousa AMM, Lopes CET, Pontes FC, Oliveira FCS, Teixeira MN, Paixão JE, Barbosa MIS, Alves VJP. Educação física na atenção primária à saúde em Sobral-Ceará: desenhando saberes e fazeres integralizados. Sanare 2009; 8(2):63-72.

24. Souza SCD, Loch MR. Intervenção do profissional de educação física nos Núcleos de Apoio à Saúde da Família em municípios do norte do Paraná. Rev Bras Ativ Fís Saúde 2012; 16(1):5-10.

25. Mendonça BCDA, Toscano JJDO, Oliveira ACCD. Do diagnóstico à ação: experiências em promoção da atividade física programa Academia da Cidade Aracaju: promovendo saúde por meio da atividade física. Rev Bras Ativ Fís Saúde 2012; 14(3):211-216.

26. Hallal PC, Tenório MCM, Tassitano, RM, Reis RS, Carvalho YM, Cruz DKA, Damascena W, Malta DC. Avaliação do programa de promoção da atividade física Academia da Cidade de Recife, Pernambuco, Brasil: percepções de usuários e não-usuários. Cad Saude Publica 2010; 26(1):70-78.

27. Hallal PC, Carvalho YM, Tassitano RM, Tenório MCM, Warschauer M, Reis RS, Cruz DKA, Damascena W, Tassitano R. Avaliação quali-quantitativa do programa Academia da Cidade, Recife (PE): concepções dos professores. Rev Bras Ativ Fís Saúde 2012; 14(1):9-14.

28. Bagrichevsky M, Castiel LD, Vasconcellos-Silva PR, Estevão A. Discursos sobre comportamento de risco à saúde e a moralização da vida cotidiana. Cien Saude Colet 2010; 15(Supl. 1):S1699-S1708.

29. Carvalho YM, Rovigatti PA, Trevizan AA. Formação em Educação Física no Brasil: outros modos de pensar e intervir no serviço público de saúde. Educ Fís Cienc 2013; 15(1):1-6. 
30. Carvalho FFB. Análise crítica da carta brasileira de prevenção integrada na área da saúde na perspectiva da Educação Física através do enfoque radical de promoção da saúde. Saúde Soc 2009; 18(1):227-236.

31. Hallal PC, Dumith SC, Bastos JP, Reichert FF, Siqueira FV, Azevedo MR. Evolução da pesquisa epidemiológica em atividade física no Brasil: revisão sistemática. Rev Saude Publica 2007; 41(3):453-460.

32. Carvalho YM. O mito atividade física/saúde [dissertação]. Campinas: Faculdade de Educação Física; 1993.

33. Castiel LD, Guilam MCR, Ferreira MS. Correndo o risco: uma introdução aos riscos em saúde. Rio de Janeiro: Editora Fiocruz; 2010.

34. Lazzarotti Filho A, Silva AM, Antunes PC, Silva APS, Leite JO. O termo práticas corporais na literatura científica brasileira e sua repercussão no campo da educação física. Movimento 2010; 16(1):11-29.

35. Damico JGS, Knuth AG. O des(encontro) das práticas corporais e atividade física: hibridizações e borramentos no campo da saúde. Movimento 2014; 20(1):329350.

36. Westphal MF. Promoção da saúde e prevenção de doenças. In: Campos GWS, Minayo MCS, Akerman M, Drumond Júnior M, Carvalho YM, organizadores. Tratado de saúde coletiva. São Paulo, Rio de Janeiro: Hucitec, Ed. Fiocruz; 2006. p. 635-667.

37. Carvalho SR, Gastaldo, D. Promoção à saúde e empoderamento: uma reflexão a partir das perspectivas crítico-social pós-estruturalista. Cien Saude Colet 2008; 13(Supl. 2):S2029-S2040.

38. Scabar TG, Pelicioni AF, Pelicioni MCF. Atuação do profissional de Educação Física no Sistema Único de Saúde: uma análise a partir da Política Nacional de Promoção da Saúde e das Diretrizes do Núcleo de Apoio à Saúde da Família - NASF. J Health Sci Inst 2012; 30(4):411-418.

39. Anjos TC, Duarte ACGO. A Educação Física e a estratégia de saúde da família: formação e atuação profissional. Physis 2009; 19(4):1127-1144.

40. Fraga $\mathrm{AB}$, Wachs $\mathrm{F}$, organizadores. Educação física $e$ saúde coletiva: políticas de formação e perspectivas de intervenção. Porto Alegre: UFRGS; 2007.

41. Czeresnia D. Epidemiologia, Ciências Sociais, integração das ciências. Rev Saude Publica 2008; 42(6):11121117.

42. Silva TS, Carvalho FFB. Promoção da saúde na atenção primária: possibilidades e desafios. In: Kalinowski CE, Figueiredo KC, Fonseca RMGS, organizadores. PROENF Programa de Atualização em Enfermagem: Atenção Primária e Saúde da Família: Ciclo 2. Porto Alegre: Artmed Panamericana; 2014. p. 71-92.

43. Alves FS, Carvalho YM. Práticas corporais e grande saúde: um encontro possível. Movimento 2010; 16(4):229-244.

Artigo apresentado em 05/02/2016

Aprovado em 21/03/2016

Versão final apresentada em 23/03/2016 